\title{
3 ADHS und Bindung
}

\author{
Ulrike Schulze
}

Auch wenn bisher keine direkte Kausalität zwischen hochunsicherer Bindung und der Manifestation eines ADHS nachgewiesen werden kann, ist davon auszugehen, dass im Verlauf der Störung die primäre Bindungsperson als wichtige externe Regulationshilfe gesehen werden kann. Das häufig bereits früh beobachtbare „schwierige Temperament" der betroffenen Kinder stellt in diesem Zusammenhang einen wichtigen Ansatzpunkt für präventive Interventionen dar. Darüber hinaus bleibt zu beachten, dass unverarbeitete mütterliche Trauer und Traumaerfahrungen Auswirkungen auf die kindliche Bindungsqualität haben.

\subsection{Bindung}

Der entwicklungspsychologische Begriff der Bindung bezeichnet ein genetisch vorgeprägtes Verhalten von Kindern, das auf die Hinwendung zu nahe stehenden Bezugspersonen ausgerichtet ist. Kinder erhalten dadurch Schutz und Versorgung. Im Falle einer ausgewogenen Bindungs-Explorations-Balance (Nähe- und Distanzregulation) kann die Bindungsperson als sichere Basis im Sine einer Quelle emotionaler Sicherheit und externe Hilfe zur Regulation wahrgenommen werden. Dies impliziert die elterliche Fähigkeit, mental die Perspektive des Kindes einzunehmen und damit in angemessener, feinfühliger Weise auf kindliche Bedürfnisse einzugehen. Bindungsrelevante Signale können also dann nur schwer adäquat beantwortet werden, wenn auf Elternseite unvollständige, gefilterte oder verzerrte Repräsentationen der essentiellen kindlichen Bedürfnisse vorhanden sind.

Mit Hilfe der sog. „Fremden Situation“ (Strange Situation Test), einem qualitativen Untersuchungsverfahren, gelang es der US-amerikanischen Entwick- 
lungspsychologin Mary Ainsworth, das Bindungsmodell John Bowlbys (Bowlby 1969) in einer standardisierten Situation beobachtbar zu machen (Ainsworth et al. 1991). Diese ermöglicht die Aktivierung kindlichen Bindungs- und Explorationsverhaltens im Rahmen einer annähernd natürlichen Situation sowie die Zuordnung der Verhaltensbeobachtungen zum jeweiligen kindlichen Bindungstyp:
A: unsicher-vermeidend,
B: sicher,
C: unsicher-ambivalent,
D: hochunsicher-desorganisiert.

Dieser wiederum muss in starker Abhängigkeit vom Verhalten der Bezugsperson gesehen werden.

Frühe Bindungssicherheit führt im Falle des Vorhandenseins weiterer wesentlicher Grundvoraussetzungen zu einer gelungenen sozial-emotionalen Entwicklung.

Im Jugend- und Erwachsenenalter kommt es über gelebte Erfahrung und hieraus entstandene sog. innere Arbeitsmodelle und die mentale Repräsentation von Bindung zur Ausbildung individueller Unterschiede in der BindungsOrganisation. Hierdurch sind (z. B. über die Auswertung des Adult Attachment Interviews, eines standardisierten Untersuchungsverfahrens zur Bindungsrepräsentation im Erwachsenenalter; Main \& Goldwyn 1998) folgende Zuordnungen möglich:

- sicher-autonom (Typ F Typ B),

- unsicher-distanziert (Typ Ds Typ A) und

- unsicher-verwickelt (Typ E Typ C).

Darüber hinaus sind hier die Bindungstypen „U“ (unresolved - unverarbeiteter Trauerprozess/nicht verarbeitetes Trauma) und CC („cannot classify“ - nicht klassifzierbar) für das Erwachsenenalter aufzuführen. Insgesamt kann in dieser Altersstufe von bewusstem und unbewusstem Wissen über Bindung, einer engen Verknüpfung von Kognitionen und Affekten sowie vorhandenen inneren Modellen vom Selbst und von relevanten Anderen ausgegangen werden.

\subsection{Neurobiologische Befunde}

„The self-organization of the developing brain occurs in the relationship with another self, another brain“ (Shore 2005). Stark vereinfacht ausgedrückt können positive Bindungsefahrungen als ein Produkt geglückter „,right brain-toright-brain-communications" aufgefasst werden.

Grundlage hierfür sind eine „kontingente Responsivität" und Synchronizität der Interaktion zwischen Kind und primärer Bindungsperson sowie das Ineinanderwirken physiologischer Rhythmen (arousal-regulation transactions). Dies impliziert das Vorhandensein elterlicher (bzw. mütterlicher) Feinfühligkeit (Fähigkeit zur Regulation eigener - auch negativer - Affekte, 
Vorhersehbarkeit von Reaktionen) im Sinne einer externen Modulationshilfe. Hierdurch wiederum kann eine Beeinflussung der für die Selbstregulation mit wesentlichen sich entwickelnden limbischen und Kortexarealen erfolgen und somit die Grundlage schaffen für die Ausbildung interner Bindungsrepräsentationen und späterer sozialer Anpassungsleistungen. Auch eine Einbeziehung weiterer Bezugspersonen ist auf dieser Basis möglich.

Wie Tierversuche zeigen, ziehen pränatale Stress-Ereignisse erhebliche Langzeiteffekte im Hinblick auf die Stressantworten auf Verhaltens- und neuroendokriner Ebene im Sinne einer „epigenetischen Programmierung“ nach sich: Aufzuführen sind hier vor allem eine langfristige Hyperaktivierung der HPA-Antwort verbunden mit einem veränderten Tag-Nacht-Rhythmus der Corstisolsekretion, jedoch auch eine mögliche Beeinträchtigung der Schlafqualität sowie der affektiven Befindlichkeit bis ins Erwachsenenalter(Darnaudéry \& Maccari 2008). Übertragen auf den Menschen und die ersten Lebensmonate ist also durchaus nachvollziehbar, dass im Falle des Aufeinandertreffens einer spezifischen Vulnerabilität mit negativen frühen Beziehungserfahrungen (z. B. Vernachlässigung, Misshandlung) über die Aktivierung der HPA-Axe eine Sensibilisierung „depressiver Bahnen“ induziert werden kann. Die Reifung des Gehirns ist demnach erfahrungsabhängig, Bindungsverhalten und Umweltfaktoren tragen zur Modifikation der Genexpression und Nervenzellfunktionen bei. Im Umkehrschluss kann dem Vorhandensein einer sicheren Bindung eine Pufferwirkung auf das sich entwickelnde Gehirn und hinsichtlich der späteren psychischen Stabilität im Sinne einer erhöhten Resilienz zugeschrieben werden (Beatson und Taryan 2003, Kaffmann \& Meany 2007).

Auch wenn postnatal von Langzeitauswirkungen wesentlicher Bindungserfahrungen auf die mentale Stabilität und Gesundheit auszugehen ist, muss grundsätzlich berücksichtigt werden, dass das Gehirn eines Neugeborenen nicht mit der unreifen Version eines „Erwachsenen“gehirns zu verwechseln, sondern zunächst unbedingt auf die Ausbildung von Bindung ausgelegt ist („Bindung als biologisches Grundbedürfnis“).

Wie aus Tiermodellen ersichtlich, wird über die Hyper-Funktion des noradrenergen Locus coerulus (LC) das Erlernen einer schnellen und robusten Präferenz für die primäre Bindungsperson über die Ausbildung einer Geruchspräferenz (Bulbus olfactorius) sowie somatosensorischen Prägung (saugen) möglich. Aufgrund der gleichzeitigen Hypo-Funktion der Amygdala kann eine erlernte Aversion gegenüber der Bindungsperson (Aufbau von Bindung auch im Falle von Misshandlungserfahrungen bzw. Traumatisierung) vermieden werden (Moriceau \& Sullivan 2005, Sullivan 2003). Dieser Befund erfährt Erweiterung durch experimentelle Beobachtungen an einer Gruppe von Rhesusaffen. Diese konnten zeigen, dass die Funktion der Amgygdala zwar nicht in fundamentalen Aspekten sozialen Verhaltens per se zu sehen ist, jedoch für das Erkennen potenziell gefährlicher Situationen und die Koordination entsprechender Verhaltensantworten als wesentlich erachtet werden muss (Bauman et al. 2004).

Aktuelle Befunde zeigen, dass dem Erkennen des eigenen Kindes und dessen bindungsrelevanter Stress-Signale bzw. deren Beantwortung spezifische 
mütterliche Hirnareale zugeordnet werden können (Noriuchi et al. 2008). Umgekehrt lassen erste Ergebnisse von Bildgebungsstudien eine Aktivierung unterschiedlicher Hirnareale bei der Betrachtung vertrauter (Mutter - enger Freund) oder aber fremder Personen sowie die Möglichkeit der topographischen Zuordnung gradueller Abstufungen bindungsrelevanter Antworten sowie einer differenzieren Antwort im Hinblick auf unterschiedliche Bindungserfahrungen vermuten (Ramasubbu et al. 2007).

Der Crad mütterlicher Zuwendung dem Neugeborenen gegenüber hat Einfluss auf die Aktivierung eines die individuelle Stress-Reaktivität determinierenden genetischen Programms (Weaver et al. 2004). Bezogen auf die Einwirkung zeitlich begrenzter Stressreaktionen (z. B. Baden des Kindes) bedeutet dies, dass das Ausmaß der mütterlichen Feinfühligkeit direkte Auswirkungen auf die Geschwindigkeit des Absinkens eines erhöhten kindlichen Cortisolspiegels zeigt (Albers et al. 2008). Darüber hinaus konnte im Rahmen einer Untersuchung ein signifikanter Zusammenhang zwischen erhöhtem mütterlichen Cortisolausspiegel während der 30.-32. SSW und der späteren Interpretation kindlichen Verhaltens aufgezeigt werden: Je höher der Spiegel, desto stärker die Assoziation mit Berichten über eine negative Reaktivität des Kindes. Darüber hinaus wurde beobachtet, dass pränatale mütterliche Angst und Depression sich zusätzlich prädiktiv im Hinblick auf das kindliche Temperament auszuwirken vermögen (Davis et al. 2007).

\subsection{ADHS - mögliche Risikofaktoren}

An dieser Stelle eingefügt sei ein kurzer Streifzug durch ätiologische Überlegungen zum ADHS. Heritabilitätsschätzungen lassen ein 5-fach erhöhtes Erkrankungsrisiko für erstgradig Verwandte annehmen. Assoziationsstudien zu Kandidatengenen betonen vor allem eine Relevanz der Gene für spezifische dopaminerge und serotonerge Rezeptoren (siehe auch Schimmelmann et al. 2006). Molekulargenetische Befunde sprechen für die Hypothese, dass sowohl dopaminerge, als auch serotonerge und noradrenerge Kreisläufe in die Entstehung dieses komplexen Störungsbildes mit einbezogen sind (Albayrak et al. 2008). Im Hinblick auf eine mögliche Persistenz der Symptomatik gelten insbesondere die familiäre Belastung mit ADHD, das Vorliegen einer psychiatrischen Comorbidität (ODD, Angst, Genussmittelabusus ...) sowie einer psychosozialen Belastung als prädiktive Faktoren (Biederman 2005).

Perinatale Komplikationen werden ernsthaft als möglicher Risikofaktor für die Manifestation eines ADHS diskutiert. Hervorzuheben sind hier vor allem eine pränatale Alkohol- und Nikotinexposition, das Auftreten einer neonatalen Hypoxie sowie ein sehr niedriges Geburtsgewicht (Schulze und Trott 1996, Ben Amor et al. 2005, Skranes et al. 2007). Bildgebende Untersuchungen sprechen für ein gemeinsames Einwirken frontaler, temporaler und parietaler Regionen auf die für die Prozesse der Aufmerksamkeit und Verhaltensinhibition wesentlichen Systeme. Darüber hinaus beschrieben sind u.a. Dysfunk- 
tionen im Bereich der fronto-subcorticalen Bahnen, Imbalancen im dopaminergen und noradrenergen System sowie spezifische anatomische Abweichungen beschrieben (Biederman und Faraone 2002, Sowell et al. 2003).

\subsection{Weitere Risikofaktoren - das „schwierige Temperament“}

Als weiterer möglicher Risikofaktor hinsichtlich der Entstehung eines ADHS muss das sogenannte „schwierige Temperament“ des Kindes (Thomas und Chess 1977) genannt werden. Die so bezeichneten Kinder zeigen Probleme im Umgang mit fremden Menschen oder Situationen, sind nur schwer zu beruhigen und werden in ihrem Verhalten als unkooperativ, inflexibel und irritierbar beschrieben.

Es erscheint nachvollziehbar, dass die Auswirkungen solcher Merkmale im Hinblick auf die spätere mögliche Manifestation bzw. Persistenz externalisierenden Verhaltens eng an die Interaktion mit der elterlichen Wahrnehmung gebunden sind.

So konnten im Rahmen einer umfassenden klinischen Studie in jeweiliger Abhängigkeit vom kindlichen Temperament deutliche Auswirkungen des elterlichen Erziehungsstils auf das Bindungsverhalten ihrer ADHD-kranken Kinder (77\%) nachgewiesen werden. Die Eltern selbst beschrieben sich häufiger als missbilligend, kontrollierend und fordernd; die eigene Erziehungsfähigkeit wurde als eingeschränkt erlebt (Finzi-Dottan et al. 2006). Kinder mit im Vordergrund stehender Hyperaktivität zeigen höhere Scores hinsichtlich ängstlicher oder vermeidender Bindung; dieser ängstliche Bindungsstil korrelierte wiederum mit einer Verbindung von elterlicher Förderung einer übermäßigen Autonomie und der kindlichen Neigung zu leichter und intensiver emotionaler Erregbarkeit. Im Vergleich hierzu wurde ein vermeidender kindlicher Bindungsstil in Verbindung mit autonomieeinschränkenden elterlichen Praktiken gefunden. Kindliche Selbstregulationsprobleme fanden sich also verstärkt durch Bindungsunsicherheit und inadäquates elterliches Verhalten. Schlussfolgernd interpretierten die Autoren das ADHD im Sinne einer Selbst-Regulationsstörung bzw. Störung mit geringer Verhaltensinhibitionsfähigkeit (Finzi-Dottan et al. 2006).

Die elterliche Durchsetzungsfähigkeit und Warmherzigkeit ist aber auch abhängig vom Grad der möglicherweise vorhandenen elterlichen Depressivität (Gerdes et al. 2007). Eine zusätzliche mögliche Einflussgröße ist Vorhandensein weiterer Risikofaktoren wie das Bestehen einer elterlichen Suchterkrankung oder ein niedriges Einkommen (Scahill et al. 1999). Nicht zuletzt hervorzuheben sind elterliche Copingstrategien im Umgang mit kindlichen Besonderheiten und Verhaltensauffälligkeiten (McKee et al. 2004).

\subsection{Bindungsstörungen}

Es muss betont werden, dass sichere und unsichere Bindungsstrategien als normale Entwicklungsvarianten aufzufassen sind und erst die hochunsichere Bin- 
dung entwicklungspsychopathologisch diskutiert werden kann. Die Entstehung dieses Bindungstyps kann dadurch erklärt werden, dass bindungsbezogene Ängste des Kindes durch zurückweisendes oder aber ängstigendes Verhalten der Bindungsperson verstärkt werden. Während in einer ängstigenden Situation das kindliche Bedürfnis nach Nähe und Trost aktiviert ist, findet keine angemessen Beantwortung durch die Bindungsperson statt. Grund hierfür sind eine fehlende Wahrnehmung der kindlichen Bedürfnisse oder eine Fehlinterpretation kindlichen Verhaltens. Somit besteht eine Unfähigkeit der Bindungsperson, die Verstörung und kindliche Furcht zu beenden (Solomon \& George 1999). Ein um das zwei- bis dreifache erhöhtes Auftreten hochunsicherer Bindung ist bei Kindern aus klinischen und Hochrisikogruppen, die psychosozial oder entwicklungsneurologisch belastet sind, beschrieben (van IJzendoorn et al. 1999). Darüber hinaus werden überzufällig häufig Zusammenhänge zwischen unverarbeiteten Trauerprozessen von Eltern über den (frühen) Verlust einer nahe stehenden Bindungsperson und hochunsicher-desorganisierter Bindung beobachtet (van IJzendoorn 1995). Hinsichtlich eines möglichen Zusammenhanges zwischen der Prädisposition zur Ausbildung dieses Bindungstyps und einer späteren erhöhten Irritierbarkeit und Hyperaktivität ist darüber hinaus eine genetische Belastung nicht auszuschließen (Lakatos et al. 2000, 2002).

Bisher werden die Bindungsstörungsdiagnosen nach ICD-10 in der kinderund jugendpsychiatrischen Praxis fast ausschließlich auf schwer vernachlässigte/früh misshandelte Kinder angewandt. Sie sind per definitionem und im Sinne einer kategorialen Einordnung als klinisch relevante und extreme Abweichungen im Bindungsverhalten aufzufassen. Während bei der Reaktiven Bindungsstörung (F 94.1; insbesondere bei jüngeren Kindern) eine Hemmung von Bindungsverhalten beobachtbar ist (keine Nähe- und Kontaktsuche bei einer Bezugsperson unter Belastung), offenbaren Kinder mit einer Bindungsstörung mit Enthemmung (F 94.2; meist Entwicklung aus F 94.1 im fünften Lebensjahr) eine relative Überaktivität des Bindungssystems; sie wirken deutlich distanzgemindert und sind nicht in der Lage, differenziertes Bindungsverhalten gegenüber einer Bezugsperson zu zeigen.

\subsection{Symptomüberschneidungen}

Bezug nehmend auf die Kernsymptomatik des ADHS lassen sich demzufolge zumindest partiell Überlappungen mit Symptomen bindungsgestörter Kinder feststellen.

So könnten expansive Verhaltensauffälligkeiten auch als mögliches Ergebnis der Anpassung an eine sozial bedrohliche Umgebung (siehe auch du Bois 2007) oder von Dysregulationen einer bestehenden Beziehungsdynamik im Sinne einer Abweichung vom Gleichgewicht zwischen Bindungs- und Erkundungsbedürfnissen interpretiert werden.

Verbindungen zur Distanzlosigkeit im Falle einer Bindungsstörung mit Enthemmung sind hier durchaus zu ziehen. 
Das teilweise massiv selbstgefährdende Risikoverhalten Kindern und Jugendlichen mit ADHS könnte aus einer (zurückliegenden) nicht ausreichenden Verfügbarkeit der Bindungsperson als sichere Basis mit erklärbar sein (Lieberman \& Zeanah 1995).

Die Beschreibung der reaktiven Bindungsstörung schließt das Vorhandensein von Aggressionen sich selbst und anderen gegenüber als Reaktion auf das eigene Unglücklichsein mit ein. Darüber hinaus werden in den Diagnoseleitlinien Einschränkungen in der Interaktion mit Gleichaltrigen und im sozialen Spiel aufgeführt.

\subsection{Comorbide Angststörungen}

Hinsichtlich der psychiatrischen Comorbidität des ADHS spielen neben oppositionellen und depressiven Störungen vor allem Angsterkrankungen bei annähernd $20 \%$ der Kinder und Jugendlichen mit ADHS eine wichtige Rolle (Elia et al. 2008).

Angststörungen zählen zu den häufigsten psychiatrischen Erkrankungen im Kindes- und Jugendalter und zeigen eine relative Symptomstabilität. Angstzustände können definiert werden als „überflutende Emotionen“, die durch das Individuum nicht einfach reguliert werden können.

Im Rahmen einer Langzeitstudie erarbeiteten Bosquet und Egeland (2006) eindrücklich ein multivariates, longitudinales Entstehungsmodell von Angststörungen von der Neugeborenenzeit bis in die Adoleszenz: 155 Hochrisikokinder wurden mittels standardisierter Verfahren über mehrere Jahre und Untersuchungszeitpunkte begleitet. Die prädiktive Bedeutung bindungsrelevanter Faktoren im Hinblick auf die Entstehung und Aufrechterhaltung von Angstsymptomen und ihre Bedeutung (Auswirkungen auf die Entwicklung der sozialen Kompetenz, Schulerfolge, Peerbeziehungen; Entstehung des Selbstbildes im Zusammenhang mit kognitiv-affektiven Repräsentationen) ließen sich bestätigen. So beeinflussten die Bindungsgeschichte und deren Biophysiologie schon zu einem frühen Zeitpunkt die weitere Regulationsfähigkeit des Kindes. Das Zusammenspiel einer vorhandenen Vulnerabilität im Sinne eines „schwierigen“ Temperaments mit gehemmtem Verhalten zeigte insbesondere im Jugendalter Auswirkungen auf die jeweilige biologisch determinierte erhöhte physiologische Reaktivität.

\subsection{ADHS und hochunsichere Bindung}

Im Rahmen einer prospektiven kontrollierten Studie nach vorangegangener Totgeburt ein Jahr post partum bzw. während des letzten Schwangerschaftstrimenons (Phase 1) und 6 bis 8 Jahre später (Phase 2) konnten Pinto et al. (2006) einen Zusammenhang zwischen desorganisierter Bindung und der späteren Beurteilung von ADHD-Symptomen durch den jeweiligen Lehrer nachweisen. Dennoch ließ sich die Diagnose einer hochunsicheren Bindung nicht als prä- 
diktiver Faktor für ein späteres ADHD herausarbeiten. Ein zurückliegendes, jedoch unverarbeitetes mütterliches Träume schien immerhin prädiktiv im Hinblick auf ein mögliches kindliches ADHD; hierbei kam die Frage auf, ob ein entsprechendes mütterliches ADHD-Rating im Zusammenspiel mit ängstigender mütterlicher Dissoziation im Falle eines schwierigen und ansprüchlichen kindlichen Verhaltens nicht eher zur Interpretation desselben als ADHD-artig geführt haben könnte.

Eine weitere Untersuchung durch Green et al. (2007) zeigte anhand einer Cruppe von 4-9-Jährigen Kindern mit externalisierenden Verhaltensauffälligkeiten eine hohe Prävalenz desorganisierter Bindung unabhängig vom Bestehen eines ADHS. Die Desorganisiertheit war jedoch assoziiert mit einem anormalen mütterlichen Ausdruck von Gefühlen (typical maternal expressed emotion).

\subsection{Vorläufige Schlussfolgerungen}

Auch wenn kein direkter kausaler Zusammenhang zwischen der kindlichen (und damit auch elterlich/mütterlichen) Bindungsgeschichte und der Manifestation eines ADHS gezogen werden kann, findet sich zumindest im Hinblick auf die Zuschreibung von Symptomen oder deren Ausprägungsrad und Persistenz eine Verbindungslinie.

Dies gilt sicherlich insbesondere im Zusammenwirken mit einer möglichen psychiatrischen Comorbidität.

Im elterlichen Umgang mit Besonderheiten ist hier auf das Vorhandensein einer ausreichenden (mütterlichen) Feinfühligkeit hinzuweisen. Diese beeinflusst die kindliche Regulationsfähigkeit nicht nur während der ersten Lebensmonate. Biographisch erworbene Grundüberzeugungen (Selbstbild, interne Repräsentationen) beeinflussen die Entwicklung eigener Copingstrategien. Somit kann hier ein wichtiger präventiver Ansatz aufgezeigt werden, der insbesondere im Zusammenhang mit dem möglichen Vorhandensein eines kindlichen „schwierigen Temperaments“ bzw. im Umgang mit der Kernsymptomatik des ADHS zum Tragen kommen sollte.

Hierbei sollte ein besonderes Augenmerk auf die psychische Befindlichkeit der primären Bezugsperson(en) (ggf. Vorhandensein eines unverarbeiteten mütterlichen Traumas, einer Suchtproblematik, depressiver Symptome, eines eigenen Aufmerksamkeitsdefizits, einer eigenen erhöhten Impulsivität) gerichtet werden. Diese sollte(n) im Hinblick auf den Umgang mit ihrem „besonderen Kind" sowie die weitere Beziehungsgestaltung und damit auch kindliche Prognose möglichst frühzeitig Beratung und (ggf. therapeutische) Begleitung erfahren. 


\section{Literatur}

Ainsworth M, Bell S, Stayton, D. Individual differences in strange situation behavior of one-year olds. In H. Scheffer (Ed.): The origins of human social relations. 1971. London: Academic Press.

Albayrak 0, Friedel S, Schimmelmann BG, Hinney A, Hebebrand J. Genetic aspects in attention-deficit/hyperactivity disorder. Journal of Neural Transmission 2008; 115(2): 305-315.

Bauman MD, Lavenex P, Mason WA, Capitanio IP, Amaral DG. The Development of Mother-Infant-Interactions after Neonatal Amygdala Lesions in Rhesus Monkeys. The Journal of Neuroscience 2004; 24 (3): 711-721.

Beatson J, Taryan S. Predisposition to depression: the role of attachment. The Australian and New Zealand Journal of Psychiatry 2003; 37 (2): 219-225.

Ben Amor L, Grizenko N, Schwartz G, Lageix P, Baron C, Ter-Stepanian M, Zappitelli M, Mbekou V, Joober R. Perinatal complications in children with attention-deficit hyperactivity disorder and their unaffected siblings. Journal of Psychiatry and Neurosciences 2005; 30(2):120-126.

Biederman I. Attention-deficit/hyperactivity disorder: a selective overview. Biological Psychiatry 2005; 57 (11): 1215-1220.

Biederman |, Faraone SV. Current concepts on the neurobiology of Attention-Deficit/Hyperactivity Disorder. Journal of Attention Disorders 2002; 6:57-16.

Bowlby I. Attachment and loss. Vol. 1: Attachment. 1969. New York: Basic Books.

Bosquet $\mathrm{M}$, Egeland $\mathrm{B}$. The development and maintenance of anxiety symptoms from infancy through adolescence in a longitudinal sample. Development and Psychopathology 2006; 18 (2): 517-550.

Darnaudéry M, Maccari S. Epigenetic programming of the stress response in male and female rats by prenatal restraint stress. Brain Research Reviews 2008; 57: 571-85.

Davis EP, Glynn LM, Dunkel Schetter C, Hobel C, Chicz-Demet A, Sandman CA. Prenatal Exposure to Matgernal Depression and Cortisol Influences Infant Temperament. Journal of the American Academy of Child and Adolescent Psychiatry 2007; 46 (6): 737-746.

du Bois R. Psychoanalytische Modelle zur Entstehung, Verarbeitung und Behandlung des ADHD. Praxis der Kinderpsychologie und Kinderpsychiatrie 2007; 56 (4): 300-309.

Elia |, Ambrosini P, Berrettini W. ADHD Characteristics: I. Concurrent Co-morbidity Patterns in Children \& Adolescents. Child and Adolescent Psychiatry Mental Health. 2008 (im Druck).

Finzi-Dottan R, Manor I, Tyano S. ADHD, Temperament, and Parental Style as Predictors of the Child's Attachment Patterns. Child Psychology and Human Development 2006; 37: 103-117.

Gerdes AC, Hoza B, Arnold LE, Hinshaw SP, Wells KC, Hechtman L, Greenhill LL, Swanson JM, Pelham WE, Wigal T. Child and parent predictors of perceptions of parent-child relationship quality. Journal of Attention Disorders 2007; 11(1):37-48.

Green I, Stanley C, Peters S. Disorganized attachment representation and atypical parenting in young school age children with externalizing disorder. Attachment and Human Development 2007; 9 (3): 207-222.

Kaffman A, Meaney J. Neurodevelopmental sequelae of postnatal maternal care in rodents: clinical and research implications of molecular insights. Journal of Child Psychology and psychiatry 2007; 48: 224-244.

Lakatos K, Toth I, Nemoda Z, Ney K, Sasvari Szekely M, Gervai J. Dopamine D4 receptor (DRD4) gene polymorphism is associated with attachment disorganisation in infants. Molecular Psychiatry 2000; 5: 633-637.

Lakatos K, Nemoda Z, Toth I, Ronai Z, Ney K, Sasvari Szekely M, Gervai J. Further evidence of the role of the dopamine, DRD4 gene in attachment ddisorganization. Interaction of the exon III $48 \mathrm{~b}$ repeat and the -521 C/T promoter polymorphisms. Molecular Psychiatry 2002; 7: 27-31.

Lieberman AF, Zeanah CH 1995. Disorders of attachment in infancy. Child and Adolescent Psychiatric Clinics of North America 1995; 4: 571-687.

Main M, Goldwyn R. Adult attachment soring and classification systems. Manual in draft: Version 6.3. may, 1998 C.C. Berkley. To be published in M. Main (Ed.). Assesing attachment through discourse, drawings, and reunion situations (working title). 1998. Cambridge: Cambridge University Press.

McKee TE, Harvey E, Danforth IS, Ulaszek WR, Friedman JL. The relation between parental coping styles and parent-child interactions before and after treatment for children with ADHD and oppositional behavior. Journal of Clinical Child and Adolescent Psychology 2004; 33 (1): 158-168. 


\section{ADHS und Bindung}

Noriuchi M, Kikuchi Y, Atsushi S. The Functional Neuroanatomy of Maternal Love: Mother's Response to Infant's Attachment Behaviors. Biological Psychiatry 2008; 63: 415-423.

Pinto C, Turton C, Hughes P, White S, Gillberg C. ADHD and Infant Disorganized Attachment. Journal of Attention Disorders 2006; 10 (1): 83-91.

Ramasubbu R, Masalovich S, Peltier S, Holtzheimer PE, Heim C, Mayberg HS. Neural Representation of Maternal Face Processing: A Functional Magnetic Resonance Imaging Study. La Revue canadienne de psychiatrie 2007; 52 (11): 726-734.

Scahill L, Schwab-Stone M, Merikangas KR, Leckman JF, Zhang H, Kasl S. Psychosocial and clinical correlates of ADHD in a community sample of school-age children. Journal of the American Academy of Child and Adolescent Psychiatry 1999; 38 (8): 976-984.

Schimmelmann BG, Friedel S, Christiansen H, Dempfle A, Hinney A, Hebebrand J. Genetische Befunde bei der Aufmerksamkeitsdefizit- und Hyperaktivitätsstörung. Zeitschrift für Kinder- und Jugendpsychiatrie und Psychotherapie 2006; 34(6): 425-433.

Schulze U, Trott G-E. Perinatale Komplikationen bei Kindern mit Hyperkinetischem Syndrom. Häufigkeit und Spezifität. Pädiatrische Praxis1996; 50 (3): 383-393.

Shore A.N. Attachment, Affect Regulation, and the Developing Brain: Linking Developmental Neuroscience to Pediatrics. Pediatrics in Review 2005; 26 (6): 204-211.

Solomon I, George $C$. The place of disorganization in attachment theory: Linking classic observations with comtemporary findings. In: Solomon J, George C (Eds.). Attachment disorganization 1999; New York: Guilford.

Skranes J, Vangberg TR, Kulseng S, Indredavik MS, Evensen KA, Martinussen M, Dale AM, Haraldseth 0, Brubakk AM. Clinical findings and white matter abnormalities seen on diffusion tensor imaging in adolescents with very low birth weight. Brain 2007; 130: 654-666.

Sowell ER, Thompson PM, Welcome SE, Henkenius AL, Toga AW, Peterson BS. Cortical abnormalities in children and adolescents with attention-deficit hyperactivity disorder. Lancet 2003; 22 (362):1699-1707.

Sullivan RM. Developing a Sense of Safety: The Neurobiology of Neonatal Attachment. Annals of the New York Academy of Sciences 2003; 1008: 122-131.

Thomas A, Chess S. Temperament and development. 1977; New York: Brunner.

van I|zendoorn MH. Adult attachment representations, parental responsiveness, and infant attachment: A meta-analysis on the predictive validity of the Adult Attachment Interview. Psychological Bulletin 1995; 117: 381-403.

van IJzendoorn MH, Schuengel C, Bakermans-Kranenburg MJ. Disorganized attachment in early childhood: Meta-analysis of precursors, concomitants and sequelae. Development and Psychopathology 1999; 11: 225-249.

Weaver IC, Cervoni N, Champagne FA, D‘Alessio AC, Sharma S, Seckl JR, Dymov S, Szyf M, Meaney M). Epigenetic programming by maternal behavior. Nature Neuroscience 2004; 7 (8): 847-854. 